\title{
Association between Depression, Daytime Sleepiness, and Thyroid Function in Patients with Thyroid Hormone Replacement Therapy
}

\author{
Jin Woo Oh', So-Jin Lee ${ }^{1,2}$, Bong-Jo Kim,2, Cheol-Soon Lee ${ }^{2,3}$, Boseok Cha' ${ }^{1,2}$, Dongyun Lee ${ }^{2,3}$, Ji-Yeong Seo ${ }^{3}$, \\ Young-Ji Lee ${ }^{3}$, Jae-Hon Lee ${ }^{1}$, Youn Jung Lee ${ }^{1}$, Myung-Hee Kang ${ }^{4,5}$, Soo Kyoung Kim, ${ }^{4,6}$, and Jae-Won Choi ${ }^{1}$ \\ Departments of ${ }^{1}$ Psychiatry, ${ }^{6}$ Internal Medicine, Gyeongsang National University Hospital, Jinju, Korea \\ Departments of ${ }^{2}$ Psychiatry, ${ }^{4}$ Internal Medicine, Gyeongsang National University College of Medicine, Jinju, Korea \\ Departments of ${ }^{3}$ Ssychiatry, Internal Medicine, Gyeongsang National University Changwon Hospital, Changwon, Korea
}

\begin{abstract}
Objective: Thyroid disease may be associated with various psychiatric disorders, such as mild depression and anxiety. Here, we examined the association between thyroid hormone levels and depression and excessive daytime sleepiness (EDS). Methods: Participants were patients who visited Gyeongsang National University Hospital for thyroid hormone replacement therapy due to chronic autoimmune thyroiditis or thyroidectomy after diagnosis of thyroid cancer between October 2012 and May 2015. Thyroid function was measured in all patients, who were also evaluated for depression, anxiety, daytime sleepiness, and resilience using the Hospital Anxiety and Depression Scale, Epworth Sleepiness Scale, and Connor-Davidson Resilience Scale. Results: The patient group consisted of 39 cases of primary hypothyroidism due to chronic autoimmune thyroiditis, especially Hashimoto's thyroiditis, and 57 cases due to total thyroidectomy after thyroid cancer. The mean ages for the participants were $52.32 \pm 5.99$ years. In patients with hypothyroidism due to chronic autoimmune thyroiditis or thyroid cancer, triiodothyronine (T3) $(\beta=0.291, p=0.013)$ was associated with depression. Both T3 $(\beta=-0.351, p=0.003)$ and free thyroxine $(\beta=-0.346, p=$ 0.008) were associated with EDS. Conclusion: We found that depression and EDS may be associated with T3 levels in patients with hypothyroidism receiving thyroid hormone replacement therapy. Therefore, we suggest that there may be a need to consider T3 levels in patients with thyroid hormone replacement therapy who complain of depression or EDS.
\end{abstract}

Key Words: Depression; Anxiety; Psychological resilience; Epworth Sleepiness Scale; Hypothyroidism

Received: June 25, 2020 Revised: July 24, 2020 Accepted: July 26, 2020

Corresponding author: Jae-Won Choi, MD, Department of Psychiatry, Gyeongsang National University Hospital, 79 Gangnam-ro, Jinju 52727, Korea.

Tel: 82-55-750-9788, Fax: 82-55-759-0003, E-mail: lingker77@naver.com

Corresponding author: Soo Kyoung Kim, MD, PhD, Department of Internal Medicine, Gyeongsang National University Hospital, Gyeongsang National University College of Medicine, 79 Gangnam-ro, Jinju 52727, Korea.

Tel: 82-55-750-8874, Fax: 82-55-759-0003, E-mail: 9854008@naver.com

(a) This is an Open Access article distributed under the terms of the Creative Commons Attribution Non-Commercial License (https://creativecommons.org/licenses/by$\mathrm{nc} / 4.0)$ which permits unrestricted non-commercial use, distribution, and reproduction in any medium, provided the original work is properly cited.

\section{INTRODUCTION}

Thyroid disease may be associated with a variety of psychiatric disorders, including mild depression and anxiety [1]. In previous studies, patients with thyroid dysfunction had a higher prevalence of mood and anxiety disorders compared to other psychiatric disorders [2]. Studies have also found that $20.5 \%$ of patients with hypothyroidism have depression, while anxiety disorders have been identified in 30-40\% patients with acute hypothyroidism [3-5].

The association between depression and thyroid hormones has been previously examined. Patients with clinically significant de- pression have been found to have lower basal thyroid stimulation hormone (TSH) [6] and decreased triiodothyronine (T3) levels [1]; however, whether excessive daytime sleepiness (EDS) was associated with either thyroid hormone in patients with hypothyroidism remains unknown. There have also been several studies that described the association between anxiety and hypothyroidism $[3,4,7,8]$. Finally, an association between depression and resilience was found in early studies [9]; however, these studies did not focus on patients with hypothyroidism.

Most patients with hypothyroidism complain of persistent fatigue and fatigue-related symptoms, despite appropriate replacement 
treatments [10,11]. Notably, fatigue is one of the most frequently reported symptoms in patients with hypothyroidism, thereby impairing quality of life $[12,13]$; however, it is difficult to distinguish fatigue and EDS due to symptom overlap [14]. In addition, EDS is common in patients with subclinical hypothyroidism [15]. Thus, we hypothesized that some patients with hypothyroidism who complain of fatigue may present with EDS. Additionally, we hypothesized that EDS may have a significant association with the levels of specific thyroid hormones such as T3, free thyroxine (fT4), and TSH.

We examined the association of several thyroid hormones with depression in patients with hypothyroidism receiving thyroid hormone replacement therapy due to thyroidectomy after diagnosis of thyroid cancer or chronic autoimmune thyroiditis. Autoimmune thyroiditis is the most common origin of primary hypothyroidism [16], in addition to irradiation or surgery [17]. Thus, we chose the autoimmune thyroiditis as the cause of primary hypothyroidism in the current study. We hypothesized that depression might be associated with hypothyroidism, and T3 or TSH levels may be associated with depression when controlling for anxiety and resilience.

\section{METHODS}

\section{Study population}

In the present study, we collected data from patients aged 20 years or older, who visited Gyeongsang National University Hospital for thyroid hormone replacement therapy due to chronic autoimmune thyroiditis or thyroidectomy after diagnosis of thyroid cancer between October 2012 and May 2015. Trained doctors evaluated and enrolled the patients in the clinic. Enrolled patients completed a questionnaire through an interview with a trained doctor in the clinic, and the questionnaire items included many variables, such as age, sex, whether or not the participants exercised, and alcohol consumption. The patient group consisted of 39 cases of primary hypothyroidism due to chronic autoimmune thyroiditis, especially Hashimoto's thyroiditis, and 57 cases due to total thyroidectomy after thyroid cancer. All participants maintained constant doses of levothyroxine (LT4) for at least 6 months before study enrollment. Thyroidectomized patients for thyroid cancer had no evidence of recurrence for more than 1 year, based on the American Thyroid Association guidelines for thyroid nodules and differentiated cancer [18]. The following individuals were excluded: pregnant women; patients with chronic comorbidities including cardiac, renal, pulmonary dysfunction, or other primary cancers; and patients with thyroid cancer who had distant metastasis. The protocol was approved by the Institutional Review Board at Gyeongsang National University Hospital (approval no. 2017-04-008). Patients provided informed written consent for participation in the study; they were not enrolled if they did not want to participate in this study. The researcher reviewed the patients' charts to confirm their clinical characteristics.

\section{Thyroid function tests}

Thyroid function was assessed in all patients at the time of enrollment. Serum TSH was measured using an immunoradiometric assay kit (Beckman Coulter, Brea, CA, USA), and T3 and fT4 were measured using radioimmunoassay kits (Beckman Coulter). The coefficients of variation were TSH $\leq 3.7 \%$, T3 $\leq 6.3 \%$, fT 4 $\leq 10.3 \%$, and free T3 $\leq 6.8 \%$. Euthyroid status was defined as TSH levels between 0.51 and $5.00 \mathrm{mIU} / \mathrm{L}$ with levels of T3 (60-200 $\mathrm{ng} / \mathrm{dL})$ and $\mathrm{fT} 4(0.70-1.80 \mathrm{ng} / \mathrm{dL})$ in reference ranges.

\section{Questionnaires for psychological well-being}

\section{Hospital Anxiety and Depression Scale questionnaire (HADS)}

To evaluate anxiety and depressive symptoms, the HADS was originally developed to assess hospital patients. The tool is divided into two scales, HADS-Depression (HADS-D) and HADS-Anxiety (HADS-A), with seven items for each scale. Each item is rated on a 4-point Likert scale; the total score for each scale ranges from $0-21$, with higher scores indicating higher levels of anxiety and depression. In our present study, the Korean version of the HADS was used to evaluate anxiety and depression and had a confirmed internal validity of Cronbach's $\alpha=0.89$. We used a cut-off score of 8 for both the HADS-A and HADS-D [19].

\section{Epworth Sleepiness Scale (ESS)}

The ESS is a self-rated tool designed to assess sleep propensity in patients who suffer from EDS. The conception of the tool originated in observation of symptoms of daytime sleepiness. The ESS has questions regarding eight different situations, and participants are asked to score how tired they would be in each situation. The total score of ESS is the sum of each score in the eight questions (from $0-3$ points) and ranges from $0-24$. A total score between $0-10$ indicates normal daytime sleepiness, while a score from 11-24 indicates a high level of daytime sleepiness [20,21]. In our current study, we used the Korean version of the ESS (K-ESS). The K-ESS demonstrated good internal consistency (Cronbach's $\alpha=0.90$ ) and testretest reliability $(\mathrm{r}=0.78-0.93)$; as such, it is a valid and reliable tool for identifying patients with EDS in Korea. The cut-off score for EDS is greater than 10 on the K-ESS [22].

\section{Connor-Davidson Resilience Scale (CD-RISC)}

The CD-RISC is a self-reported questionnaire consisting of 25 items. It has been used to evaluate coping skills and psychological resilience. Each item is rated on a 5-point Likert scale, with a higher total score indicative of greater psychological resilience. In previous studies, great validity (Cronbach's $\alpha>0.9$ ) and reliability (Pearson $\mathrm{r}=0.7$ ) of the Korean version of the CD-RISC (K-CD-RISC) has been well-established across various groups. The 2010 version of the K-CD-RISC was used to evaluate resilience in the current study $[9,23]$. 
Statistical analysis

Demographic characteristics, such as sex and age of hypothyroidism, as well as whether or not the participants exercise, and alcohol consumption are presented in Table 1. Continuous variables, such as HAM-D, HAM-A, ESS, are presented as mean and standard deviation. Chi-squared tests were performed to compare categorical variables. Each variable had a few missing data; thus, we analyzed each variable by using data except missing values in the demographic table. In addition, multiple linear regression analyses were performed to identify predictors of depression or EDS. We performed multiple linear regression models to determine which thyroid hormone was associated with depression when controlling for age, sex, thyroid cancer/chronic autoimmune thyroiditis, L-thyroxine (Synthroid) dosage, anxiety, resilience, and daytime sleepiness in patients with hypothyroidism. The relationship between anxiety and depression has been examined in many previous studies [24]; thus, we included anxiety as a confound. We performed additional multiple linear regression models to determine how TSH, T3, and fT4 correlate with EDS after controlling for age, sex, thyroid cancer/autoimmune thyroiditis, L-thyroxine dosage, anxiety, and depression in patients with hypothyroidism receiving thyroid hormone replacement therapy. We used SPSS (version 25; IBM Corp., Armonk, NY, USA) for the statistical analyses. A twotailed $\mathrm{p}$-value $<0.05$ was considered statistically significant.

Table 1. Sociodemographic and clinical characteristics of patients with hypothyroidism receiving thyroid hormone replacement therapy

\begin{tabular}{lc}
\multicolumn{1}{c}{ Characteristics } & Values \\
Sex $(\mathrm{n}=96)$ & \\
Male & $15(15.6)$ \\
Female & $81(84.4)$ \\
Age $(\mathrm{yr})(\mathrm{n}=96)$ & $52.32 \pm 5.99$ \\
Exercise $(\mathrm{n}=94)$ & \\
Yes & $53(56.4)$ \\
No & $41(43.6)$ \\
Alcohol (n=95) & \\
Yes & $35(36.8)$ \\
No & $60(63.2)$ \\
Hypothyroidism (n=96) & \\
Thyroid cancer & $39(40.6)$ \\
Chronic autoimmune thyroiditis & $57(59.4)$ \\
L-thyroxine $(m g)(n=96)$ & $0.125 \pm 0.065$ \\
T3 (ng/dL) (n=83) & $95.0 \pm 18.3$ \\
TSH (mIU/L) (n=95) & $2.4 \pm 7.5$ \\
fT4 (ng/dL) (n=95) & $1.5 \pm 0.3$ \\
HADS-A (n=93) & $4.8 \pm 2.9$ \\
HADS-D (n=92) & $5.3 \pm 3.1$ \\
ESS ( $\mathrm{n}=91)$ & $7.6 \pm 3.4$ \\
CD-RISC (n=90) & $27.5 \pm 7.1$ \\
\hline Data a $p$ res & \\
\hline
\end{tabular}

Data are presented as $n(\%)$ or mean \pm SD. HADS-A: Hospital Anxiety and Depression Scale-Anxiety, HADS-D: Hospital Anxiety and Depression Scale-Depression, ESS: Epworth Sleepiness Scale, CD-RISC: the Connor-Davidson Resilience Scale, T3: triiodothyronine, TSH: thyroid-stimulating hormone, fT4: free thyroxine

\section{RESULTS}

The participants were patients with hypothyroidism, including hypothyroidism due to thyroid cancer $(\mathrm{n}=57)$ or chronic autoimmune thyroiditis $(\mathrm{n}=39)$. The demographics and clinical status are presented in Table 1 . The mean ages for the participants were 52.32 \pm 5.99 years. Overall, $28 \%(\mathrm{n}=26)$ demonstrated clinical depression measured by HADS-D, 20\% $(\mathrm{n}=19)$ showed clinical anxiety measured by HADS-A, and $29 \%(\mathrm{n}=26)$ showed EDS ( $\mathrm{ESS} \geq 10$ ).

Multiple linear regression analyses were performed to determine predictors of depression when controlling for age, sex, thyroid cancer/chronic autoimmune thyroiditis, anxiety, daytime sleepiness, and resilience (Table 2). T3 ( $\beta=0.291, \mathrm{p}=0.013$ ) was associated with depression (adjusted $\mathrm{R}^{2}=0.213$ ). Additional multiple linear regression analyses were performed to identify the predictors of EDS when controlling for age, sex, and thyroid cancer/chronic autoimmune thyroiditis, depression, and anxiety (Table 3). Both T3 level $(\beta=$ $-0.351, \mathrm{p}=0.003$, adjusted $\left.\mathrm{R}^{2}=0.167\right)$ and $\mathrm{fT} 4(\beta=-0.346, \mathrm{p}=0.008$, adjusted $\mathrm{R}^{2}=0.148$ ) were associated with EDS (Table 3 ).

\section{DISCUSSION}

This study demonstrated that $\mathrm{T} 3$ levels were associated with depression in patients with hypothyroidism due to autoimmune thyroiditis or thyroid cancer when controlling for anxiety, daytime sleepiness, and resilience. In addition, T3 and fT4 levels were significantly correlated with EDS in patients with hypothyroidism; however, depression was not found to be associated with EDS, which is inconsistent with previous studies [25].

Table 2. Multiple linear regression analysis for the association between thyroid function and depression of patients with hypothyroidism

\begin{tabular}{cccccc}
$\begin{array}{c}\text { Independent } \\
\text { variable }\end{array}$ & $\mathrm{B}$ & $\mathrm{SE}$ & $\beta$ & $\mathrm{T}$ & $\mathrm{p}$ \\
TSH & 0.034 & 0.044 & 0.087 & 0.777 & 0.440 \\
$\mathrm{~T} 3$ & 0.049 & 0.019 & 0.291 & 2.545 & 0.013 \\
fT4 & 1.420 & 1.276 & 0.144 & 1.113 & 0.269 \\
\hline
\end{tabular}

Adjusted for age, sex, thyroid cancer/chronic autoimmune thyroiditis, L-thyroxine, HADS-A, CD-RISC, and ESS. HADS-A: Hospital Anxiety and Depression Scale-Anxiety, CD-RISC: the Connor-Davidson Resilience Scale, ESS: Epworth Sleepiness Scale, T3: triiodothyronine, TSH: thyroid-stimulating hormone, fT4: free thyroxine

Table 3. Multiple linear regression analysis for the association between thyroid function and daytime sleepiness of patients with hypothyroidism

\begin{tabular}{cccccc}
$\begin{array}{c}\text { Independent } \\
\text { variable }\end{array}$ & $\mathrm{B}$ & $\mathrm{SE}$ & $\beta$ & $\mathrm{T}$ & $\mathrm{p}$ \\
TSH & 0.014 & 0.051 & 0.031 & 0.267 & 0.790 \\
T3 & -0.066 & 0.021 & -0.351 & -3.076 & 0.003 \\
fT4 & -3.640 & 1.332 & -0.346 & -2.733 & 0.008 \\
\hline
\end{tabular}

Adjusted for age, sex, thyroid cancer/chronic autoimmune thyroiditis, L-thyroxine, HADS-A, and HADS-D. HADS-A: Hospital Anxiety and Depression Scale-Anxiety, HADS-D: Hospital Anxiety and Depression Scale-Depression, T3: triiodothyronine, TSH: thyroid-stimulating hormone, fT4: free thyroxine 
We found that higher T3 levels might be used to predict depressive symptoms in patients with hypothyroidism receiving thyroid hormone replacement therapy. Previous studies have reported that most patients who were depressed with thyroid dysfunction have decreased T3 levels [1]. In patients with hypothyroidism who were treated with thyroid hormone replacement therapy and had fT4 and TSH levels return to normal, depression may worsen as T3 levels increase. Thus, clinicians might check thyroid function for abnormally high T3 levels when patients who are treated for hypothyroidism present with a depressed mood.

The association between EDS and thyroid hormones, such as T3, fT4, and TSH, has not previously been demonstrated. Furthermore, no studies have identified a hormone factor that might predict EDS. In the present study, patients with hypothyroidism demonstrated rescued TSH and fT4 levels through thyroid hormone replacement therapy. We found that lower T3 and fT4 levels were associated with greater daytime sleepiness. A previous study reported that $13 \%$ of patients with subclinical hypothyroidism complained of daytime sleepiness with a score of 10 or greater on the ESS [26]. Thyroid hormones may act as a transporter with some neurotransmitters, for example, histamine and noradrenaline [27]. Additionally, the histaminergic tuberomammillary nucleus of the hypothalamus and noradrenergic locus coeruleus of the pons are dominant wakefulness-promoting nuclei and are important in autonomic regulation $[28,29]$. L-thyroxine administration has been reported to increase histamine levels in the hypothalamus and brain cortex [30]. In previous rodent studies, T3 levels were limited to brain nuclei, which receive strong noradrenergic innervation [31], and both noradrenergic centers and non-adrenergic projection sites are dense regions of T3 [27]. Therefore, alterations in T3 and/ or L-thyroxine levels might affect the arousal-promoting system. Moreover, when thyroid hormones were injected into the preoptic area of mice with impaired thyroid function, rapid eye movement (REM), non-REM, and total sleep time were shortened and arousal increased [32]. Thus, T3 and fT4 levels might activate the arousal-promoting system and be a clinically important factor for maintaining arousal in patients with hypothyroidism.

Of the various factors that were included as confounds, we found that anxiety, but not depression, was positively correlated with EDS. There have been a few reports of an association between anxiety and EDS in the general population. One study found that elevated somatic and cognitive anxiety symptoms were associated with daytime sleepiness in older adults [33]. In university students with clinical insomnia, $50.8 \%$ of the participants who had clinically severe anxiety complained of EDS versus $30.9 \%$ of controls [34]. However, no studies have found whether anxiety increases daytime sleepiness in patients with hypothyroidism. Thus, this was the first study to report that anxiety might increase EDS in patients with hypothyroidism when controlling for age, sex, thyroid cancer/autoimmune thyroiditis, L-thyroxine, depression, and anxiety.

The current study had several strengths. We were able to identify the association between thyroid hormone levels and depression or EDS. Concurrently, we included various confounds such as TSH,
L-thyroxine dosage, anxiety, and depression. Of these, L-thyroxine dosage was not included as a confound in previous studies.

We are also aware of several limitations. First, there were no control groups who did not have hypothyroidism. Therefore, we could not identify the effects of hypothyroidism on EDS, anxiety, and depression in comparison with a control group. Second, the total number of participants was small. Thus, we could not add crucial confounders including underlying diseases, sleep efficiency, and insomnia severity index when we used the multiple linear regression model. Third, because of the small number of participants, patients with hypothyroidism could not be classified into two groups, especially in the multiple linear regression model (i.e., thyroid cancer and chronic autoimmune thyroiditis). If the total number of participants was larger, patients with hypothyroidism could be divided into two groups to identify differences in the cause of hypothyroidism. To compensate for this, we considered two major causes of hypothyroidism as one categorical confounder and included it in the multiple linear regression model because the number of participants in the two groups was similar. Due to the small number of participants, this study had low adjusted $\mathrm{R}^{2}$ scores. Fourth, the current research was a cross-sectional study, so it was not possible to assess a temporal association between exposure and outcome. Hence, longitudinal studies are necessary to derive the causal relationship in future.

The present study focused on the association between T3 levels and depression when controlling for anxiety, resilience, and daytime sleepiness as confounds in patients with hypothyroidism. In addition, we found that EDS may be associated with lower T3 or fT4 levels in patients with hypothyroidism. Thus, we suggest that there might be a need to consider T3 or fT4 levels in patients with thyroid hormone replacement therapy who complain of depression or EDS.

\section{Acknowledgments}

None

\section{Conflicts of Interest}

The authors have no potential conflicts of interest to disclose.

\section{Author Contributions}

Conceptualization: So-Jin Lee, Jae-Won Choi. Data curation: Jin Woo Oh, Dongyun Lee. Formal analysis: Ji-Yeong Seo, Young-Ji Lee. Investigation: Jae-Hon Lee, Youn Jung Lee. Methodology: Myung-Hee Kang, Soo Kyoung Kim. Bong-Jo Kim, Cheol-Soon Lee. Software: Jin Woo Oh. Validation: So-Jin Lee, Boseok Cha. Writing-original draft: Jin Woo Oh, So-Jin Lee. Writing-review \& editing: Bong-Jo Kim, Cheol-Soon Lee, Boseok Cha, Dongyun Lee, Ji-Yeong Seo, Young-Ji Lee, Jae-Hon Lee, Youn Jung Lee, Myung-Hee Kang, Soo Kyoung Kim, Jae-Won Choi.

\section{ORCID iDs}

Soo Kyoung Kim (D)

https://orcid.org/0000-0002-7230-4033 
Jae-Won Choi (1)

https://orcid.org/0000-0002-4516-1954

Jin Woo Oh (1)

https://orcid.org/0000-0003-0960-7549

\section{REFERENCES}

1. Hage MP, Azar ST. The link between thyroid function and depression. J Thyroid Res 2012;2012:590648.

2. Placidi GP, Boldrini M, Patronelli A, Fiore E, Chiovato L, Perugi G, et al. Prevalence of psychiatric disorders in thyroid diseased patients. Neuropsychobiology 1998;38:222-225.

3. Hall RCW. Psychiatric effects of thyroid hormone disturbance: psychosomatic illness review: no. 5 in a series. Psychosomatics 1983;24:7-18.

4. Haug TT, Mykletun A, Dahl AA. The association between anxiety, depression, and somatic symptoms in a large population: the HUNT-II study. Psychosom Med 2004;66:845-851.

5. Gupta S, Saha PK, Mukhopadhyay A. Prevalence of hypothyroidism and importance of cholesterol estimation in patients suffering from major depressive disorder. J Indian Med Assoc 2008;106:240-242.

6. Poirier MF, Lôo H, Galinowski A, Bourdel MC, Rémi-Bouissière P, Piketty $\mathrm{ML}$, et al. Sensitive assay of thyroid stimulating hormone in depressed patients. Psychiatry Res 1995;57:41-48.

7. Whybrow PC, Prange AJ Jr, Treadway CR. Mental changes accompanying thyroid gland dysfunction. A reappraisal using objective psychological measurement. Arch Gen Psychiatry 1969;20:48-63.

8. Haggerty JJ Jr, Garbutt JC, Evans DL, Golden RN, Pedersen C, Simon JS, et al. Subclinical hypothyroidism: a review of neuropsychiatric aspects. Int J Psychiatry Med 1990;20:193-208.

9. Jung YE, Min JA, Shin AY, Han SY, Lee KU, Kim TS, et al. The Korean version of the Connor-Davidson Resilience Scale: an extended validation. Stress Health 2012;28:319-326.

10. Saravanan P, Chau WF, Roberts N, Vedhara K, Greenwood R, Dayan CM. Psychological well-being in patients on 'adequate' doses of 1-thyroxine: results of a large, controlled community-based questionnaire study. Clin Endocrinol (Oxf) 2002;57:577-585.

11. Wekking EM, Appelhof BC, Fliers E, Schene AH, Huyser J, Tijssen JG, et al. Cognitive functioning and well-being in euthyroid patients on thyroxine replacement therapy for primary hypothyroidism. Eur J Endocrinol 2005; 153:747-753.

12. Jaeschke R, Guyatt G, Cook D, Harper S, Gerstein HC. Spectrum of quality of life impairment in hypothyroidism. Qual Life Res 1994;3:323-327.

13. Watt T, Hegedüs L, Rasmussen AK, Groenvold M, Bonnema SJ, Bjorner JB, et al. Which domains of thyroid-related quality of life are most relevant? Patients and clinicians provide complementary perspectives. Thyroid 2007; 17:647-654.

14. Pigeon WR, Sateia MJ, Ferguson RJ. Distinguishing between excessive daytime sleepiness and fatigue: toward improved detection and treatment. J Psychosom Res 2003;54:61-69.

15. Akatsu H, Ewing SK, Stefanick ML, Fink HA, Stone KL, Barrett-Connor E, et al. Association between thyroid function and objective and subjective sleep quality in older men: the Osteoporotic Fractures in Men (MrOS) study. Endocr Pract 2014;20:576-586.

16. Dayan CM, Daniels GH. Chronic autoimmune thyroiditis. N Engl J Med 1996;335:99-107.
17. Sklar C, Whitton J, Mertens A, Stovall M, Green D, Marina N, et al. Abnormalities of the thyroid in survivors of Hodgkin's disease: data from the Childhood Cancer Survivor Study. J Clin Endocrinol Metab 2000;85:3227-3232.

18. Haugen BR, Alexander EK, Bible KC, Doherty GM, Mandel SJ, Nikiforov YE, et al. 2015 American Thyroid Association Management Guidelines for adult patients with thyroid nodules and differentiated thyroid cancer: The American Thyroid Association Guidelines Task Force on thyroid nodules and differentiated thyroid cancer. Thyroid 2016;26:1-133.

19. Oh SM, Min KJ, Park DB. A study on the standardization of the Hospital Anxiety and Depression Scale for Koreans: a comparison of normal, depressed and anxious groups. J Korean Neuropsychiatr Assoc 1999;38:289296.

20. Johns MW. A new method for measuring daytime sleepiness: the Epworth Sleepiness Scale. Sleep 1991;14:540-545.

21. Johns MW. Reliability and factor analysis of the Epworth Sleepiness Scale. Sleep 1992;15:376-381.

22. Cho YW, Lee JH, Son HK, Lee SH, Shin C, Johns MW. The reliability and validity of the Korean version of the Epworth Sleepiness Scale. Sleep Breath 2011;15:377-384.

23. Baek HS, Lee KU, Joo EJ, Lee MY, Choi KS. Reliability and validity of the Korean version of the Connor-Davidson Resilience Scale. Psychiatry Investig 2010;7:109-115.

24. Runkewitz K, Kirchmann H, Strauss B. Anxiety and depression in primary care patients: predictors of symptom severity and developmental correlates. J Psychosom Res 2006;60:445-453.

25. Hein M, Lanquart JP, Loas G, Hubain P, Linkowski P. Prevalence and risk factors of excessive daytime sleepiness in major depression: a study with 703 individuals referred for polysomnography. J Affect Disord 2019;243:2332.

26. Shinno H, Ishikawa I, Yamanaka M, Usui A, Danjo S, Inami Y, et al. Effect of levothyroxine on prolonged nocturnal sleep time and excessive daytime somnolence in patients with idiopathic hypersomnia. Sleep Med 2011;12: 578-583.

27. Dratman MB, Gordon JT. Thyroid hormones as neurotransmitters. Thyroid 1996;6:639-647.

28. Steininger TL, Alam MN, Gong H, Szymusiak R, McGinty D. Sleep-waking discharge of neurons in the posterior lateral hypothalamus of the albino rat. Brain Res 1999;840:138-147.

29. Wu MF, Gulyani SA, Yau E, Mignot E, Phan B, Siegel JM. Locus coeruleus neurons: cessation of activity during cataplexy. Neuroscience 1999;91:13891399.

30. Upadhyaya L, Agrawal JK. Effect of L-thyroxine and carbimazole on brain biogenic amines and amino acids in rats. Endocr Res 1993;19:87-99.

31. Dratman MB, Futaesaku Y, Crutchfield FL, Berman N, Payne B, Sar M, et al. Iodine-125-labeled triiodothyronine in rat brain: evidence for localization in discrete neural systems. Science 1982;215:309-312.

32. Moffett SX, Giannopoulos PF, James TD, Martin JV. Effects of acute microinjections of thyroid hormone to the preoptic region of hypothyroid adult male rats on sleep, motor activity and body temperature. Brain Res 2013; 1516:55-65.

33. Gould CE, Spira AP, Liou-Johnson V, Cassidy-Eagle E, Kawai M, Mashal N, et al. Association of anxiety symptom clusters with sleep quality and daytime sleepiness. J Gerontol B Psychol Sci Soc Sci 2018;73:413-420.

34. Choueiry N, Salamoun T, Jabbour H, El Osta N, Hajj A, Rabbaa Khabbaz L. Insomnia and relationship with anxiety in university students: a cross-sectional designed study. PLoS One 2016;11:e0149643. 